\title{
Hallucinations and parkinsonian motor fluctuations
}

\author{
W. Fernandez, G. Stern and A. J. Lees \\ University College and Middlesex Hospital School of Medicine, Department of Neurology, \\ Mortimer Street, London W1N 8AA, UK \\ Correspondence to: A.J. Lees, The National Hospital for Neurology and Neurosurgery, \\ Queen Square, London WC1N 3BG, UK
}

\begin{abstract}
Thirty patients with Parkinson's disease experiencing hallucinations during long-term treatment were compared with 20 parkinsonian patients without hallucinations. No differences were found in the duration of disease, $\mathrm{L}$-dopa treatment or disease severity between the two groups. The hallucinators however, were significantly older and more cognitively impaired. Visual hallucinations occurring only during "off periods of immobility" were relatively common and improved concurrently with parkinsonian disabilities after L-dopa. Although visual hallucinations were commonest auditory hallucinations occurred in one third of the hallucinators.
\end{abstract}

Keywords: Hallucinations - Parkinson's disease

\section{INTRODUCTION}

Visual pseudohallucinations and visual hallucinations occur commonly in patients with Parkinson's disease receiving anti-parkinsonian medication (Goetz et al., 1982). Associated depressive illness, cognitive or visual impairment are known predisposing factors (Berrios and Brook, 1984; Sandyk and Gillman, 1985; Korezyn et al., 1986) and marked neocortical involvement with Lewy body pathology is a further recognised cause (Perry et al., 1989). Cholinergic deficiency has also been proposed as a pathogenetic factor (Perry et al., 1985, 1990; Dubois et al., 1990).

The relationship between the appearance of hallucinations and the motor state of patients with L-dopa related on-off fluctuations has not, however been well studied although both on and off period psychiatric side-effects are recognised (Hardie et al., 1984; Menza et al., 1990). We have examined this issue and also the relationship between depression and dementia and the occurrence of hallucinations.

\section{PATIENTS AND METHODS}

Patients were randomly sampled from the population of parkinsonian patients attending the Movement disorder clinics at the Middlesex and University College Hospital, and the National Hospital for Neurology and Neurosurgery, London. All selected cases fulfilled the UK-P.D.S. brain bank criteria for Parkinson's disease (Gibb and Lees, 1988) and their drug history was recorded. The patients with additional help from relatives and carers were then asked to establish whether or not they had experienced hallucinations, and if so detailed descriptions were obtained.

The patients were divided into those who had experienced hallucinations and those who had not. Dementia and depression were assessed using the mini-mental test (Folstein et al., 1975) and DSM-III-R criteria (APA, 1987), respectively. The relationship between the mental and motor states, in particular relation to simultaneous fluctuations in motor and mental disabilities were also studied. The two groups of patients were compared using Student's $t$-test for paired data.

\section{RESULTS}

Fifty patients were included in the study. Forty-two had on-off fluctuations. Thirty patients had hallucinations and 20 patients denied ever experiencing them. The general features of these two populations of patients are shown in Table I. There were no statistically significant differences between hallucinators and non-hallucinators in terms of the type or duration of parkinsonian symptoms, severity of the disease as judged by Hoehn-Yahr scale, duration of motor fluctuations, L-dopa treatment duration or L-dopa dose.

However significant differences were found between the two groups when looking at age $(p<0.001)$, and degree of cognitive deterioration $(p<0.001)$. Elderly patients were much more prone to develop hallucinations. 
TABLE I. Clinical features of 50 parkinsonian patients

\begin{tabular}{lccl}
\hline & Hallucinators & Non-hallucinators & \\
\hline Age (years) & $65+8.8$ & $54+11.5$ & $p<0.001$ \\
$\begin{array}{l}\text { Parkinsonism duration } \\
\quad \text { (years) }\end{array}$ & $12.5+5.7$ & $11.15+4.9$ & $p<0.395$ \\
On-off duration (years) & $5.0+4.3$ & $3.8+3.2$ & $p<0.27$ \\
L-Dopa duration (years) & $11.1+5.1$ & $9.2+4.7$ & $p<0.182$ \\
L-Dopa dose (mg) & $695+495$ & $731+539$ & $p<0.8$ \\
Hoehn-Yahr score & $3.6+0.5$ & $3.2+0.6$ & $p<0.1$ \\
Minimental test score & $23.9+6.3$ & $29.2+1.3$ & $p<0.001$ \\
\hline
\end{tabular}

There were 15 demented patients in the hallucinator group and none in the non-hallucinators group. The number of patients affected by depression was comparable in the two groups: six in the hallucinator group and four in the nonhallucinators group.

Nineteen of the 30 patients with hallucinations were unaware of any link between the occurrence of hallucinations and their "on" and "off" states. These patients experienced hallucinations in both motor states and in 11 they were particularly prominent in the evening and night. In eleven cases the hallucinations were related closely to motor state: Three patients had hallucinatory symptoms exclusively when mobile (on period), and eight patients were affected by hallucinations during the immobile off periods. None of the patients with selective off-period hallucinations had dementia, as judged by mini-mental scores.

The hallucinations had a clear relationship with drug intake in 14 cases. This relationship was established because of the onset or aggravation of the mental symptoms when increasing the drug dose, or improvement of the hallucinations with reduction of the dose. In five cases the hallucinations were related to bromocriptine, in three cases to L-dopa, in three cases to apomorphine, in one case to lisuride, in one case to anticholinergics and in one case to L-dopa withdrawal. There were seven patients on anticholinergic treatment in the hallucinators group and four in the non-hallucinator group. The latency to develop hallucinations after starting L-dopa treatment was an average of 9.7 years.

There was no significant difference between the reported frequency of vivid dreams and nightmares between the hallucinators (12 cases) and non-hallucinators (10 cases). The hallucinations were threatening in 18 cases. In 9 cases the patients had additional auditory hallucinations. In the single instance where they occurred as an isolated mental symptom they occured in the on period. In 29 cases the patients had visual hallucinations: Animals were seen by the patients in 14 cases and situations involving human beings were seen in 23 cases. The variety of animals reported was very wide and included spiders, rats, flies, cats, dogs, foxes, horses, parrots and squirrels. The visual hallucinations involving people consisted of relatives, children, soldiers, murderers, cripples, singers and "strange people", mainly involving women. In one case a patient imagined being in bed with his wife and another man. In those patients who had hallucinations in relationship to the motor on-off oscillations, the mental symptoms fluctuated with a very similar time period to the motor symptoms. Two clinical cases are presented to illustrate the mental symptoms encountered.

\section{Case 1}

A 54-year-old retired engineer with Parkinson's disease for 17 years and motor on-off fluctuation with dyskinesias for 13 years was admitted to the hospital for a trial of L-dopa withdrawal. Thirty-six hours after L-dopa withdrawal and when having off period disabilities because of tremor, hypokinesia and rigidity he began to hear war planes bombing central London and saw tanks inside the hospital. He reported adults and children leaving the hospital in a hurry and strange people on the wall of his room. On examination he was disorientated and his mini-mental test score was 24 . He was put on L-dopa treatment again and $48 \mathrm{~h}$ later was fully orientated, free of hallucinations and his mini-mental test score was 28 . One year after this episode the patient developed hallucinations and confusion again, predominantly at night, whilst having treatment with L-dopa and apomorphine, and he now requires small doses of chlorpromazine to overcome his mental symptoms.

\section{Case 2}

A 78-year-old man with Parkinson's disease for 21 years and severe motor fluctuations over the last 2 years, was admitted to the hospital and began treatment with apomorphine via subcutaneous infusion as well as his regular L-dopa and bromocriptine. In the early morning when "off" with bradykinesia, tremor and inability to walk, he began to talk about four strange people he saw at the window of his room who were threatening him; his apomorphine dose was increased in an attempt to overcome his motor disabilities but then he began to describe a film he was seeing on the wall of his room. He developed penile 
erections and adopted catatonic postures, with sustained flexion of his legs, knees, trunk, and neck, arms extension and blepharospasm. He described an erotic film he was seeing and began to masturbate despite bradykinesia and tremor in his right hand; this epsiode lasted for $10 \mathrm{~min}$, culminating in rhythmic pelvic movements and ejaculation. After this period of time the patient was able to walk displaying choreic movements in his trunk and arms and was free from hallucinations.

\section{DISCUSSION}

It has been generally supposed that hallucinations in parkinsonian patients occur as a side effect of drug therapy (Celesia and Barr, 1970; Sweet et al., 1976). However, in this study the dose or duration of L-dopa treatment and the duration and severity of the disease did not differentiate hallucinators and non-hallucinators. Older patients and those who had cognitive impairment were more prone to develop hallucinations. Hallucinations were reported before the use of L-dopa and anticholinergic drugs (Rondot $e t$ $a l ., 1984)$ and have been reported in patients affected by diffuse Lewy body disease (Gibb et al., 1987). These observations suggest that hallucinations might be related to the underlying pathology in the cerebral cortex in the majority of cases. Recent work has shown that Lewy bodies are present to greater or lesser degree in all cases of Parkinson's disease (Hughes et al., 1992).

Although non-threatening visual pseudohallucinations are the commonest perceptual misinterpretation seen in treated Parkinson's disease (Moskovitz, 1978) one third of our patients also had auditory hallucinations and in two thirds the visual hallucinations were terrifying.

On period-visual hallucinations due to L-dopa therapy could be due to overstimulation of the mesostriatal and mesocorticolimbic dopamine receptors. However, it is more difficult to explain the frequent occurrence of visual hallucinations in off periods. These may represent a withdrawal syndrome analogous to off-period dystonia and occur as a result of differential stimulation of dopamine receptor sub-types in the cortex.

Off-period hallucinations have occasionally been described previously (Sage and Duvoisin, 1986; Nissenbaum et al., 1987; Steiger et al., 1991) as well as during L-dopa holidays (Mayeux et al., 1985; Lang, 1987). The fact that hallucinations are most common at night, when the patients are more likely to be "off" suggests that the frequency of off period-hallucinations has been underestimated. This clinical phenomenom is of interest because it has relevance to the treatment. In patients with Parkinson's disease and hallucinations, it is crucial to try and establish whether the mental symptoms occur predominantly during the off periods, where an increase rather than a reduction in the dose of the antiparkinsonian treatment would be indicated. In similar light, although a L-dopa holiday has been recommended as a treatment for parkinsonian patients with hallucinations, one should be aware that hallucinations can occur as a result of L-dopa withdrawal.

\section{REFERENCES}

American Psychiatric Association (1987) Diagnostic and Statistical manual of Mental Disorders, 3rd Edn revised. APA, Washington, DC.

Berrios GE and Brook P (1984) Visual hallucinations and sensory delusions in the elderly. British Journal of Psychiatry, 144, 662-664.

Celesia G and Barr AN (1970) Psychosis and other psychiatric manifestations of levodopa therapy. Archives of Neurology, 23, 193-200.

Dubois B, Pillon B, Lhermitte F and Agid Y (1990) Cholinergic deficiency and frontal dysfunction in Parkinson's disease. Annals of Neurology, 28, 17-121.

Folstein MF, Folstein SE and McHugh PR (1975) "Mini-mental state". A practical method for grading the cognitive state for the clinician. Psychiatry Research, 12, 189-198.

Gibb WRG and Lees AJ. The relevance of the Lewy body to the pathogenesis of idiophatic Parkinson's disease. Journal of Neurology, Neurosurgery and Psychiatry, 51, 745-752.

Gibb WRG, Esiri MM and Lees AJ (1987) Clinical and pathological features of diffuse Lewy body disease (Lewy body dementia). Brain, 110, 131-1153.

Goetz CG, Tanner CM and Klawans HL (1982) Pharmacology of hallucinations induced by long-term drug therapy. American Journal of Psychiatry, 139, 494-497.

Hardie RJ, Lees AJ and Stern GM (1984) On-off fluctuations in Parkinson's disease. A clinical and neuropharmacological study. Brain, 107, 487-506.

Hoehn MM and Yahr MD (1967) Parkinsonism: onset, progression and mortality. Neurology, 17, 427-442.

Hughes AJ, Daniel SE and Lees AJ (1992) Archives of Neurology, in press.

Korczyn AD, Inzelberg R, Treves T, Neufeld M, Reider I and Rabey PM (1986) Dementia of Parkinson's disease. Advances in Neurology, 45, 399-403.

Lang AE (1987) Sudden confusion with levodopa withdrawal. Movement Disorders, 2, 223 (letter).

Mayeux R, Stern Y, Mulvey K and Cote L (1985) Reappraisal of temporary levodopa withdrawal ("drug Holiday") in Parkinson's disease. New England Journal Medical, 313, 724-728.

Menza MA, Sage J, Marshall E, Cody R and Duvoisin R (1990) Mood changes and "on-off" phenomena in Parkinson's disease. Movement Disorders, 5, 148-151.

Moskovitz C, Moses H and Klawans H (1978) Levodopainduced psychosis: a kindling phenomenon. American Journal of Psychiatry, 135, 669-675.

Nissenbaum H, Quinn NP, Brown RG, Toone B, Gotham AM and Marsden CD (1987) Mood swings associated with the "on-off" phenomenon in Parkinson's disease. Psychological Medicine, 17, 899-904.

Perry EK, Curtis M, Dick DJ, Candy JM, Atack JR, et al. (1985) Cholinergic correlates of cognitive impairment in Parkinson's disease: comparisons with Alzheimer's disease. Journal of Neurology, Neurosurgery and Psychiatry, 48, 413-421.

Perry EK, Kerwin J, Perry RH, Blessed G and Fairbairn AF. Vis- 
ual hallucinations and the cholinergic system in dementia. Journal of Neurology, Neurosurgery and Psychiatry, 53, 88 (letter).

Perry RH, Irving D, Blessed G, Perry EK and Fairban AF (1989) Clinically and neuropathologically distinct form of dementia in the elderly. The Lancet, $\mathbf{i}, 166$.

Rondot P, de Recondo J, Coignet A and Ziegler M (1984) Mental disorders in Parkinson's disease after treatment with L-dopa. Advances in Neurology, 40, 259-269

Sage JI and Duvoisin RC (1986) Sudden onset of confusion with severe exacerbation of Parkinsonism during levodopa therapy. Movement Disorders, 1, 267-270.

Sandyk R and Gillman MA (1985) Depression as a cause of hallucinations in Parkinson's disease. Clinical Neuropharmacology, 8, 199-200.

Steiger MJ, Quinn NP, Toone B and Marsden CD (1991) Offperiod screaming accompanying motor fluctuations in Parkinson's disease. Movement Disorders, 6, 89-90.

Sweet RD, McDowell FH, Feigenson JS, Loranger AW and Goodell H (1976) Mental symptoms in Parkinson's disease during chronic treatment with levodopa. Neurology, 26, 305-310. 


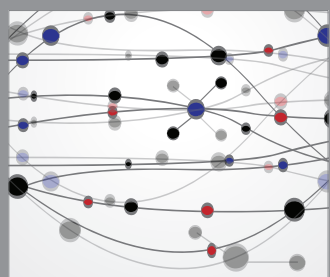

The Scientific World Journal
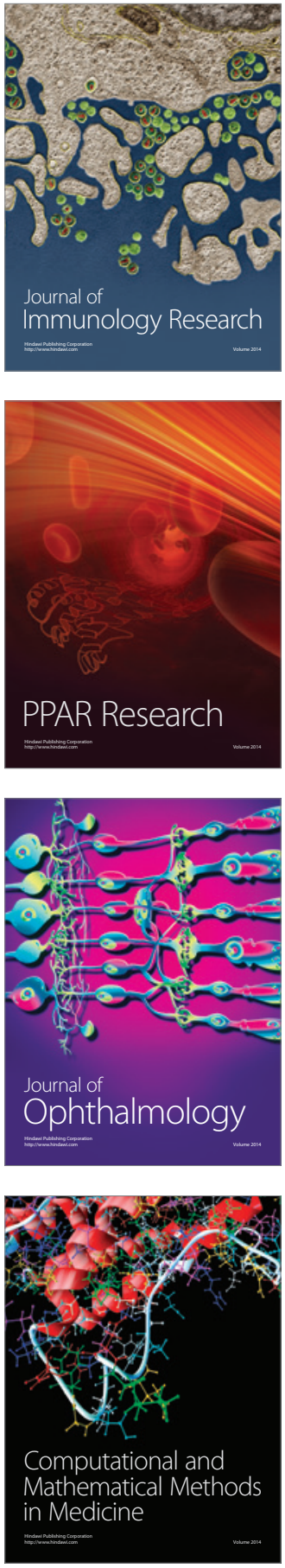

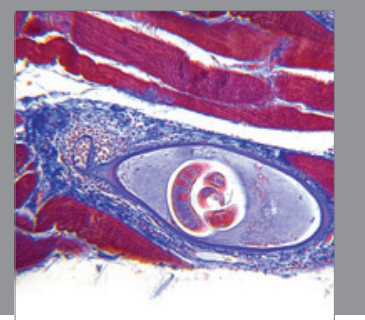

Gastroenterology

Research and Practice
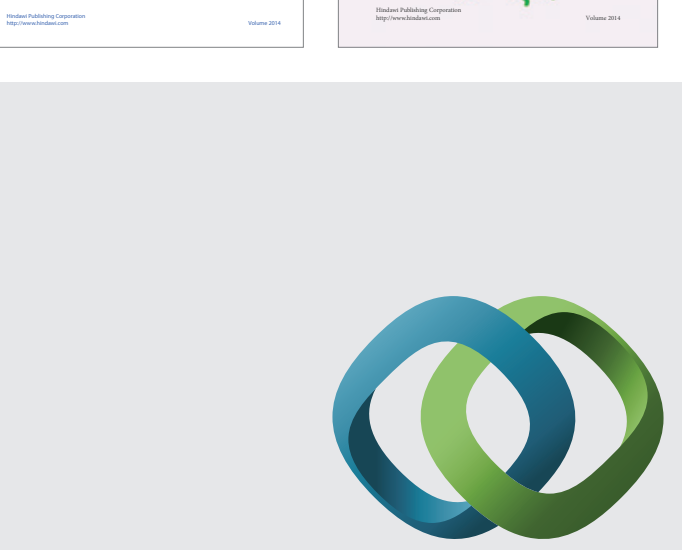

\section{Hindawi}

Submit your manuscripts at

http://www.hindawi.com
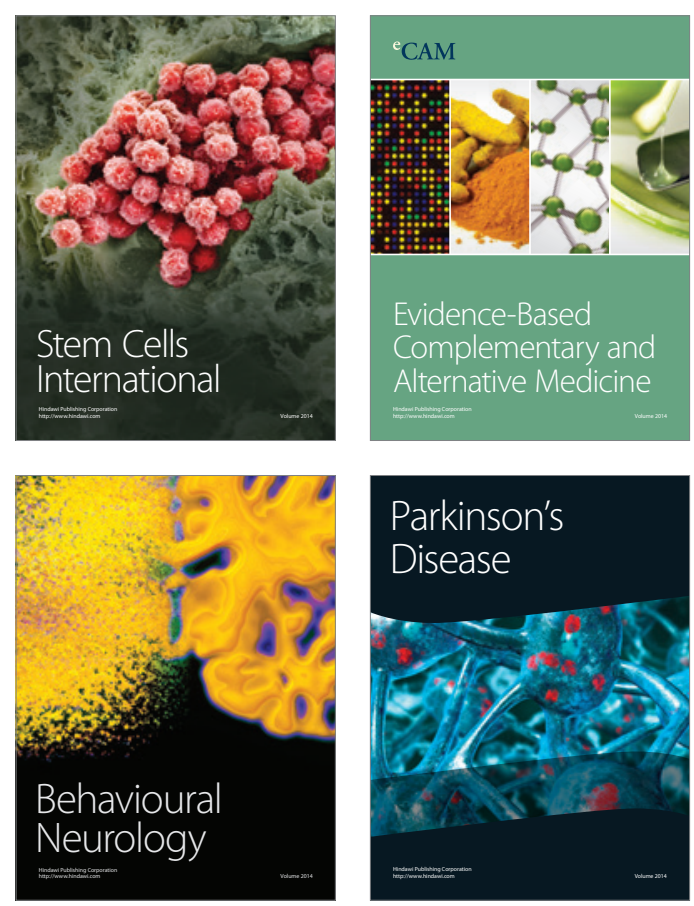

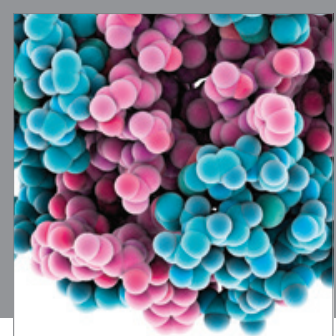

Journal of
Diabetes Research

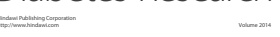

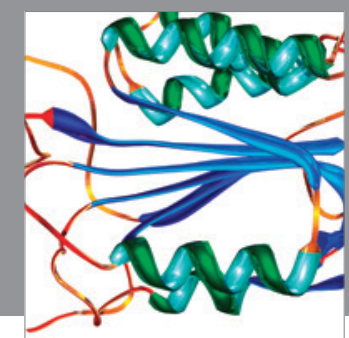

Disease Markers
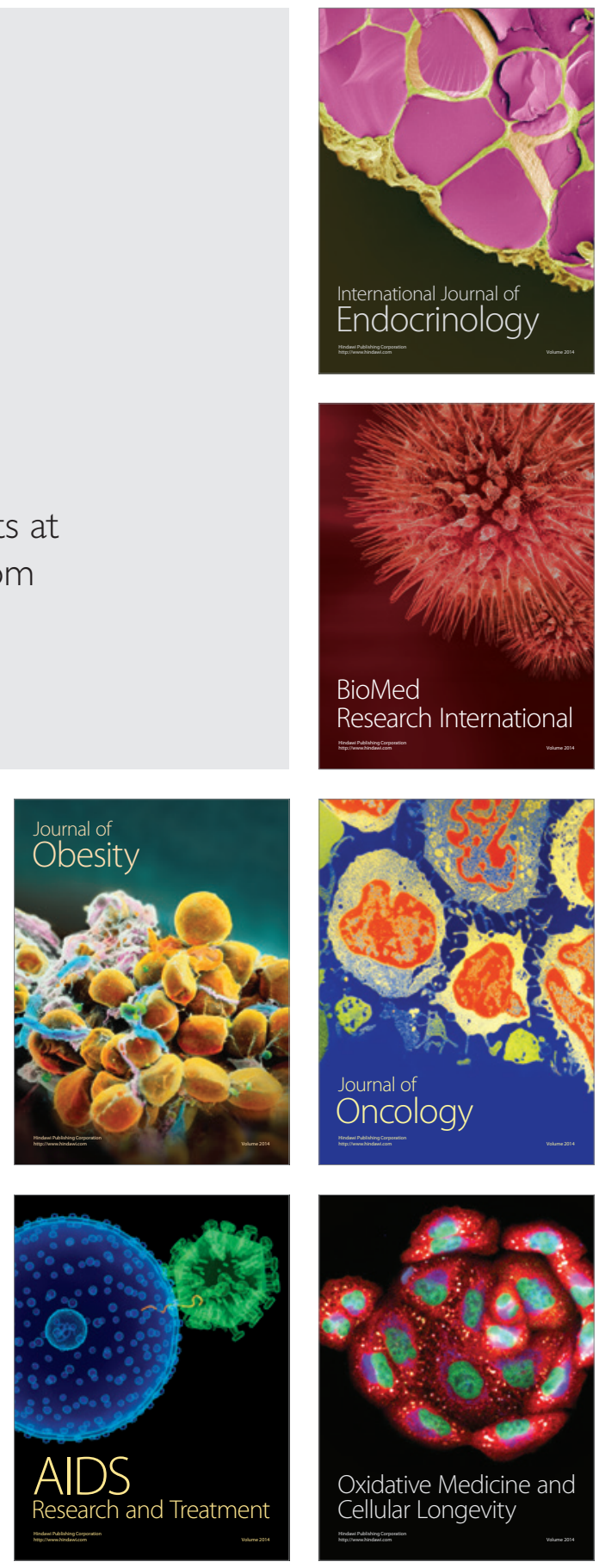